\title{
Intelligent agent-based modeling of kinesin nanomotor
}

\begin{abstract}
This paper introduces a new methodology to develop comprehensive structural and behavioral models of kinesin nanomotor within its cell using agent technology. In this work, firstly, kinesin nanomotor is introduced as a physical intelligent agent. Then, we have proposed an agent-based structural model of kinesin nanomotor using composite diagram of Unified Modeling Language (UML). We also introduced its heads and tail as the kinesin's sensors and its motor domain as its actuator. An agent-based behavioral model of kinesin nanomotor was developed using Finite State Machine (FSM) diagram of UML and illustrated the internal intelligent and autonomous decision-making process of the nanomotor. The proposed agent-based behavioral FSM model was verified with its mathematical definitions which developed as Deterministic Finite Automaton (DFA) and its respective grammar. The outputs of the proposed grammar were in agreement with the proposed DFA model and traced the behavior of the nanomotor in nature. Also, the behavioral DFA model of the nanomotor was implemented as its software agent model. The proposed agent-based structural and behavioral models of kinesin nanomotor introduced the nanomotor as bionanoagent which was able to sense its cell through its sensors, make decision internally, and perform actions upon the cell through its actuator.
\end{abstract}

